\title{
NEW GEOMETRY AND PROPERTIES OF ADVANCED SUPERIONIC CONDUCTORS
}

\author{
Alexandra ANDREEVA, Alexandr DESPOTULI \\ Institute of microelectronics technology and high purity materials Russian Academy of Sciences, \\ Chernogolovka, Russian Federation, andreeva@ipmt-hpm.ac.ru
}

https://doi.org/10.37904/nanocon.2019.8502

\begin{abstract}
Advanced superionic conductors (AdSICs) have the best ion-transport properties. These properties are determined by the AdSIC crystal structure that is close to optimal for fast ionic transport (FIT). The structure represents a close-packed cubic lattice of motionless ions penetrated by a 3D-network of uniform FIT-tunnels in which mobile ions move by hopping mechanism. The descriptors, based on a symmetry group of an immobile rigid sublattice and a local symmetry interconnected interstitial sites through which mobile ions move, are revealed. A new geometry and packing of FIT-tunnels inside the unit cell are presented. Some unusual properties of AdSICs are discussed. It is shown that the specific crystal structure of AdSICs may serve as a basis for searching and 3-D designing new solid ionic materials with high ion-transport properties.
\end{abstract}

Keywords: Advanced superionic conductors, structural descriptors, tunnels of fast ionic transport, universal dynamic response, 3-D structure design

\section{INTRODUCTION}

At extremely small sizes ionic devices for information storage and processing have a huge advantage over electronic devices concerning tunnel leakage currents which are connected with mass of charge carriers $(m)$, as $\propto 1 / \exp \left(m^{1 / 2}\right)$. Another area of perspective applications of solid-state ionic materials is impulse storages of charge and energy. The uniqueness of these devices is a high concentration of mobile ions $\left(10^{22} \mathrm{~cm}^{-3}\right)$ and a fast ionic transport (FIT). It allows realizing charge accumulation in extremely thin double layer with high current density on ionic/electronic heteroboundary. The heterojunctions are the main working parts of new devices because interfaces can often control the final performance. Both interfacial engineering and new solid-state ionic materials with FIT and low activation energy of ion movement $\left(E_{\mathrm{a}}\right)$ should be essential components for designing novel devices with high ion- transport characteristics, e.g. thin film impulse supercapacitors [1]. The purpose of this work is to present a substantiation of the term "an advanced superionic conductor" (AdSIC). The task is to reveal distinctive unique structural properties of AdSICs and to propose on this basis the ways and potentialities for searching and designing new ionic conductors and the creation of efficient devices with FIT on nanoscale.

\section{CLASSIFICATION OF IONIC CONDUCTORS. ADVANCED SUPERIONIC CONDUCTORS}

The majority of ionic solids usually have a very low ionic conductivity $\left(\sigma_{i}\right)$ at room temperature $(R T)$, usually, it is less than 1 microS/cm. However there are some families of compounds based on $\mathrm{H}^{+}, \mathrm{Li}^{+}, \mathrm{Cu}^{+}, \mathrm{Ag}^{+}, \mathrm{F}^{-}$and O-- ionic conductors which have $\sigma$ of $\sim 0,001$ to $1 \mathrm{~S} / \mathrm{cm}$ at $R T$ or moderately high temperatures (considerably lower than the melting temperatures). Among them are solid electrolytes, with $\sigma_{e}<<\sigma_{i}$ ( $\sigma_{e}$ is electronic conductivity) and mixed conductors, with noticeable $\sigma_{e}$. The classification of ionic conductors in the coordinates $\sigma_{1}-\sigma_{\mathrm{e}}$ [1-2] introduced into a field of solid-state ionics a new class of conductors AdSICs. They have the best ion-transport properties, such as a high $\sigma_{\mathrm{i}}>0.1 \mathrm{~S} / \mathrm{cm}$ at $R T$ and a low $E_{\mathrm{a}}$ about $0.1 \mathrm{eV}$. These properties are connected with AdSIC crystal structure, optimal for FIT. The $\sigma_{i}$ depends on several factors, but the most important are the heights of potential barriers $(\eta)$ which moving ions should overcome on their path 
by the hopping mechanism from one potential well to the next. The highest potential barrier on pathway of ionic transport defines value of $E_{\mathrm{a}}$. The examples of AdSICs are cubic crystals such as $\alpha-\mathrm{Agl}(\mathrm{Im} 3 \mathrm{~m})$ with $E_{\mathrm{a}}$ $\sim 0.09 \mathrm{eV}, \sigma$ is about $1.2 \mathrm{~S} / \mathrm{cm}$ at $147^{\circ} \mathrm{C}$ and compounds of $\alpha-\mathrm{RbAg}_{4} l_{5}$ family $\left(\mathrm{P} 4{ }_{132}\right)$ with record high ionic conductivity at $R T\left(\alpha-\mathrm{RbAg}_{4} I_{5}\right.$ with $\sigma_{\mathrm{i}} \sim 0.35 \mathrm{~S} / \mathrm{cm} ; \alpha-\mathrm{Rb}_{4} \mathrm{Cu}_{16} 1_{7} \mathrm{Cl}_{13}$ with $\left.\sigma_{\mathrm{i}} \sim 0.5 \mathrm{~S} / \mathrm{cm}, E_{\mathrm{a}} \approx 0.1 \mathrm{eV}\right)$.

The $\sigma-\sigma_{e}$ plot allows to distinguish a new class of «hypothetical superionic conductors» with higher $\sigma$ as compared with AdSICs. Ionic solids with such characteristics have not been found in nature, but structure fragments with "hypothetical" ion-transport characteristics are known. E.g., the studies on the structure and phase transformations in minerals of the pearceite-polybasite group with the general formula $(\mathrm{Ag}, \mathrm{Cu})_{16} \mathrm{M}_{2} \mathrm{~S}_{11}$, $(\mathrm{M}=\mathrm{Sb}, \mathrm{As})$ [3] revealed the superionic phase with a trigonal symmetry and $E_{\mathrm{a}} \sim 0.16 \mathrm{eV}$ at temperatures close to $R T$. In this phase, there are "rings" with a diameter of an order of $8 \AA$, where $\mathrm{Ag}+$ ions move with a very low $E_{a}(\sim 15 \mathrm{meV})$. Modern methods of material engineering allow manipulating processes at the molecular and atomic levels during materials synthesis, to create fundamentally new architectures, new metamaterials and structures with unique characteristics and functionality. Therefore, search for natural and creation of artificial superionic conductors $(\mathrm{SIC})$ with $E_{\mathrm{a}} \sim k T$ at $R T$ can be expected in future.

\section{STRUCTURAL FEATURES AND DESCRIPTORS OF ADSICS}

Sunandana C.S. in [4] has formulated a short formula for the SIC (or fast ionic conductor) structure:

A fast ionic conductor $=$ frame sub-lattice + partial basis + disordering

The formula emphasizes that SIC structure is described by two sub-lattices, one of which (frame), formed by motionless ions, has a certain basis, and the other, a «liquid-like» sub-lattice of mobile ions, has no basis.
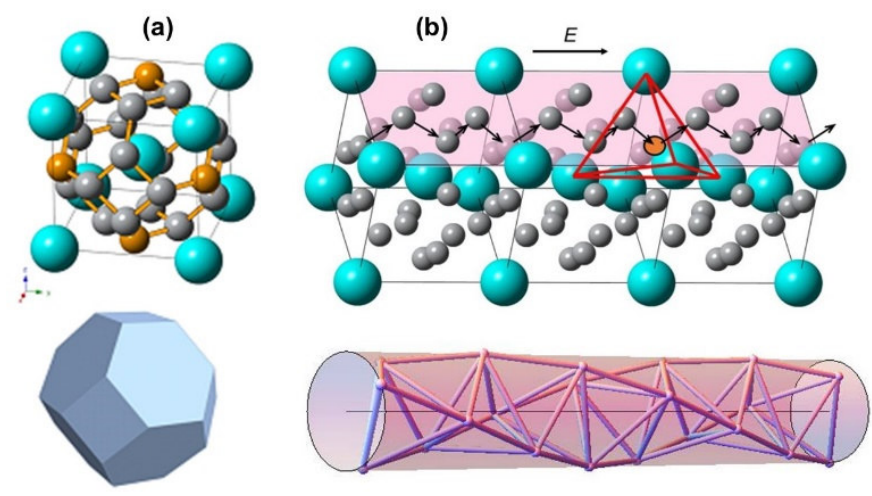

Figure 1 Structure of AdSIC $\alpha$-Agl, (a) The rigid sub-lattice of large iodine anions. Within the I--sub-lattice, there is a set of interconnected tetrahedral-interstitial sites (T-sites, little balls), which forms a 3D network of FIT- pathways in the unit cell (truncated octahedron). (b) Possible pathway (FIT-tunnel, it is shown by arrows) of mobile Ag+ ions through T-sites in external electrical field $E$. The schematic FIT- tunnel presents a chain of deformed iodine tetrahedrons conjugated by faces (one of this tetrahedrons is shown)

Solid superionic phases are formed at phase transitions when a co-operative excitation of mobile ions is initiated, the ions pass from the normal positions of initial phase to interstitial sites of new SIC phase that leads to the $E_{\mathrm{a}}$ decrease. For example, for Agl compound there are two low temperature phases $\mathrm{\gamma}$-Agl (zincblende $\mathrm{F}-432$ ) and $\beta-\mathrm{Agl}$ (wurtzite P6 ${ }_{3} \mathrm{mc}$ ) with a distinct crystal basis and without noticeable $\sigma$. The high temperature superionic phase $\alpha$-Agl has basis $(\mathrm{Im} 3 \mathrm{~m})$ only for lattice of immobile iodine ions and $\sigma$ of $\alpha$-Agl is $6-7$ orders higher than $\sigma$ of $\mathrm{Y} / \mathrm{B}-\mathrm{AgI}$ phases. As the temperature rises, the Ag-l bond becomes weaker and collapses at $147^{\circ} \mathrm{C}$, it stimulates the formation of the close packed $\alpha$-Agl phase of higher density as compared with the $\gamma / \beta$ 
- phases existing at $R T$. In $\alpha$-Agl, the iodine ions form a close packed bcc lattice, where $\mathrm{Ag}^{+}$ions occupy interstitial sites. According to neutron diffraction data [in 4], the preferential pathways for mobile $\mathrm{Ag}^{+}$-ions pass through tetrahedral interstitial sites ( $T$-sites, which are separated from each other by low potential barriers) in the cubic lattices of $\alpha-A g l$ and $\alpha-\left.R_{\text {RAg }}\right|_{5}$. In $\alpha$-Agl unit cell, the number of T-sites is 6 times larger than that of the $\mathrm{Ag}^{+}$mobile ions. These T-sites form a 3D percolation network of FIT-tunnels (truncated octahedron) for $\mathrm{Ag}^{+}$mobile ions in the unit cell of $\alpha-\mathrm{Agl}$ (Figure 1a). Figure 1b shows possible pathway of mobile Ag+ ions through T-sites in external electrical field $E$. This pathway can be presented as a net of similar deformed tetrahedrons conjugated by faces. The deformation of tetrahedron for $\alpha-\mathrm{Agl}(\operatorname{Im} 3 \mathrm{~m}, \mathrm{a}=0.5067 \mathrm{~nm})$ is $\sim 13.5 \%$ for tetrahedron edges and $|\Delta \varphi| \sim 10^{\circ}$ for tetrahedron angles. Among close-packed Bravais lattices, only bcc has the percolated net of T-sites. The $\sigma$ of $\alpha-\mathrm{Agl}$ at $147^{\circ} \mathrm{C}$ is about $1.2 \mathrm{~S} / \mathrm{cm}$ with $E_{\mathrm{a}} \sim 0.09 \mathrm{eV}$ (in $\mathrm{\gamma} / \beta-\mathrm{Agl}, E_{\mathrm{a}}$ $\sim 0.6 \mathrm{eV}$ ). The $E_{\mathrm{a}}$ of bcc structures was estimated for rigid iodine lattice depending on the radius of a mobile ion and was shown [5] that the minimum of $E_{a}$ corresponds to the Ag+-ion. The AdSICs of mixed type, such as $\alpha-\mathrm{Ag}_{2} \mathrm{~S}$ and $\alpha-\mathrm{Ag}_{2} \mathrm{Se}$ also have the bcc structure.

(a)

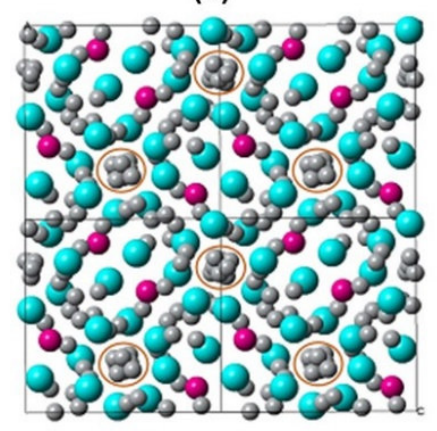

(b)

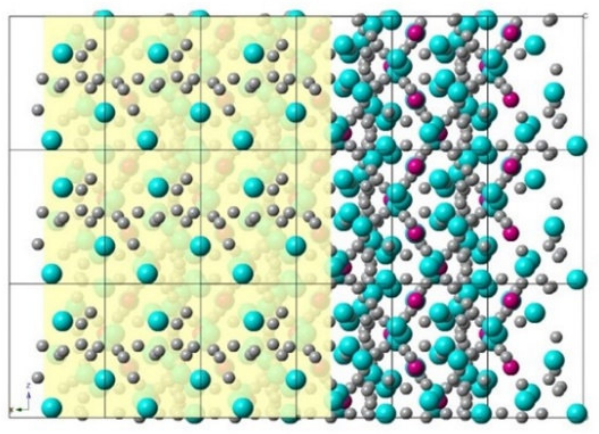

(c)

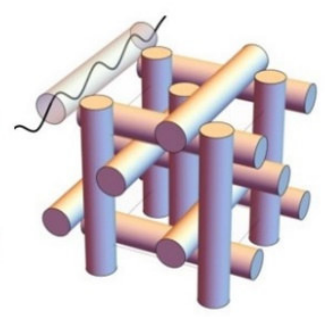

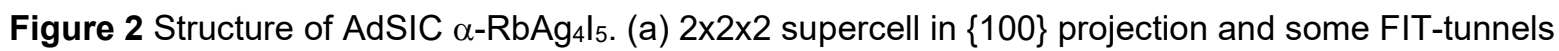
perpendicular to $\{100\}$, composed by T-sites, are marked by rings (large balls are iodine ions, middle balls are $\mathrm{Rb}^{+}$- ions and little balls are interstitial sites through which mobile $\mathrm{Ag}^{+}$- ions hop); (b) another orientation of FIT-tunnels after structure turning; (c) dense cubic 3D-packing of rods in elementary cell, where each rode represents a FIT-tunnel with low potential landscape for mobile ions movement.

The cubic structure with the space group $\mathrm{P}_{1132}$ is characteristic of AdSICs $\alpha$-RbAg4 $I_{5}$-family with record high $\sigma$ at $R T$. The arrangement of the iodine ions is similar to the arrangement of atoms in the $\beta-\mathrm{Mn}(\mathrm{P} 413)$ structure [6], whereas $\mathrm{Rb}^{+}$-ions of the rigid lattice occupy 4 (a) positions in the distorted iodine octahedrons. Within iodine frame there are 56 deformed tetrahedrons (T-sites) which are conjugated by faces, thus creating diffusion pathways (FIT- tunnel) for $\mathrm{Ag}^{+}$ions. The analysis of all possible positions of FIT-tunnels for $\alpha-\mathrm{RbAg}_{4} \mathrm{I}_{5}$ (Figure 2) leads to a new geometry, namely 3-D dense packing of rods. According analysis the basic features of AdSIC's structure are: (1) high concentration, $\sim 10^{22} \mathrm{~cm}^{-3}$, of mobile ions; (2) a rigid close-packed lattice of immobile ions inside which the mobile ions hop through interstitial crystallographic sites of definite symmetry; (3) a percolation 3D-network of interconnected interstitial low-energy sites (T-sites) which form FIT-tunnels with shallow potential landscape. It is characteristic of a cubic symmetry; (4) an optimum FIT-tunnel with low $E_{a}$ composed by atomic tetrahedrons conjugated by faces; (5) certain relations between the sizes and polarization of mobile and immobile ions in the FIT-tunnels to achieve a barriers distribution with low value of Ea.

The dense 3D packing of FIT-tunnels exists inside cubic lattice of immobile ions. The 3D network of topologically uniform FIT-tunnels formed by conjugated by faces tetrahedrons is characteristic feature of the AdSIC's unit cell. This property is the basic descriptor of Im3m ( $\alpha$-Agl) and P4132 ( $\left.\alpha-\left.\mathrm{RbAg}_{4}\right|_{5}\right)$ space groups. 


\section{THE “ANOMALY” IN FREQUENCY BEHAVIOUR OF CONDUCTIVITY IN ADSICS}

Along with unusual phase transformations AdSICs reveal a new distinctive property such as the absence of the universal dynamic response (Jonsher's law [7]), which used in materials science as standard method of the characterization of solid ionic materials. The law establishes interrelation between a real part of complex conductivity $\left(\operatorname{Re}^{*}\right)$ and frequency $(\omega)$ of external electric influence for materials with hopping conductivity.

$$
\operatorname{Re} \sigma^{*}(\omega) \propto \omega^{n}(n<\approx 1)
$$

The creation of new impulse devices with FIT demands testing methods based on the advanced theory of ionic transport on nanoscale. We have offered a new theoretical system - «structure-dynamic approach of nanoionics» (SDA) [8], which allows to make computer experiments and to describe the interconnected iontransport and dielectric-polarization processes in model SE-nanostructures. Main components of SDA are: (1) The layered model of a nanostructure with 1D-geometry of FIT tunnels, which combines a set $\left\{X^{\prime}\right\}$ of parallel crystallographic planes $X^{j}$ separated by potential barriers $\eta_{j, j+1}$. Each $X^{j}$ plane corresponds to minima in a potential landscape and normal to $X$ oriented along FIT-tunnels as well as along direction of effective electrostatic field $F_{\text {eff. }}(2)$ The method of dynamic "hidden" variables $n_{i}(t)$ which are concentrations of mobile ions on $\{X\}$ planes. (3) The physic-mathematical formalism, i.e., detailed-balance relations and a system of kinetic differential equations which operate with a set $\left\{n_{i}(t)\right\}$. (4) The method of a uniform effective electrostatic field $F_{\text {eff }}$ of $X j$ plane. (5) The new notion - "Maxwell displacement current on a potential barrier".

According to SDA, the Jonsher's law holds for solid-state ionic conductors in the frequency range depending on the heights $\eta$ and the heights difference $(\Delta \eta)$ of potential barriers in the crystal structure. At sufficiently high values of $\eta$ and $\Delta \eta$ always there are spatial areas with the local Maxwell displacement currents $I_{D j, j+1}$ and ionic currents $l_{j, j+1}$ of comparable values, that leads to the universal response. The absence of Jonsher's law in AdSIC polycrystals at $R T$ indicates that in a frequency range up to $\sim 10^{10} \mathrm{~Hz}, I_{D j}, j+1<<I_{j, j+1}$. Computer calculations have shown that frequencies on which Maxwell displacement currents $I_{D j, j+1}$ on a potential barrier become comparable or exceed ionic currents $l_{j, j+1}$ depend on barrier height $\eta$. For a barrier with energy $0.2 \mathrm{eV}$ it is $\omega$ about $10^{7} \mathrm{~Hz}$. According to [9], at $300 \mathrm{~K}$ in polycrystals of AdSIC $\alpha$-RbAg $4 I_{5}$ the Jonsher's law is absent up to frequencies $10^{9}-10^{10} \mathrm{~Hz}$. A comparison of experimental data with computer calculations leads to a conclusion, that in AdSICs heights $\eta$ are not above $0.2 \mathrm{eV}$ and $\Delta \eta \leq 0.1 \mathrm{eV}$ and the response has an ohmic character, i.e., does not depend on $\omega$. If to consider that the basic changes of potential landscape $\Delta \eta$ occur near grain boundaries (GBs), evidently, processes of structural self-organization and formation of low-energy coherent GBs for AdSICs are characteristic phenomena.

\section{ADSIC'S STRUCTURE AS A GUIDE FOR SEARCHING AND DESIGNING NEW IONIC MATERIALS}

The best ion-transport properties of AdSICs $\left(\sigma_{\mathrm{i}}>10^{-1} \mathrm{~S} / \mathrm{cm}\right.$ at $\left.300 \mathrm{~K}, E_{\mathrm{a}} \approx 0.1 \mathrm{eV}\right)$ are characteristic of space groups Im3m and P4132. The main descriptor of AdSICs is the 3D - network of homogeneous FIT-tunnels for mobile ions, existing on the level of unit cell of cubic symmetry. Close-packed structures with the hcp and fcc coordination prevail over those with the bcc structure. According to [10], the difference in $E_{a}$ for ions hopping between T-T positions in the bcc structure and between T-O-T positions in the hcp and fcc structures at $R T$ correspond to large changes of $\sigma_{i}$. Examples of rigid anionic lattices which have FIT tunnels basically with Tsites for mobile ions are scarce. High $\sigma_{i}$ of solid-state ionic conductors, i.e. a low $E_{a}$, requires along with high concentration of mobile ions a shallow energy landscape along the migration pathway (in FIT-tunnel). It is possible to assume that presence in structure of FIT-tunnels composed by crystallographic T-sites can form the basis for revealing high ion-transport properties. In $[10,11]$ the bcc lattice has been used as a descriptor to search for some new $\mathrm{Na}^{+}$and $\mathrm{Li}^{+}$- fast ionic conductors. 
The 3D-nets of ion low energy pathways play pivotal role in FIT. The more FIT- tunnels in structure, the higher $\sigma_{i}$. The higher symmetry of point group, the more symmetrically and, therefore, energetically equivalent sites can be generated. An increase of $\sigma_{i}$ in ionic systems needs to be considered in the context of structure design. The design provides additional possibilities for achievement of record high $\sigma_{i}$ at RT. Synthesis of nanostructures with a 3D network of FIT-tunnels is an important task of solid-state ionics.

(a)

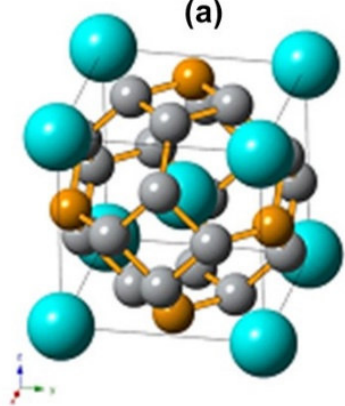

(b)

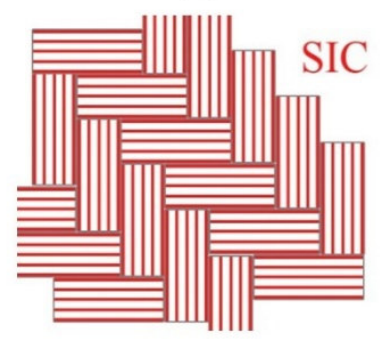

(c)

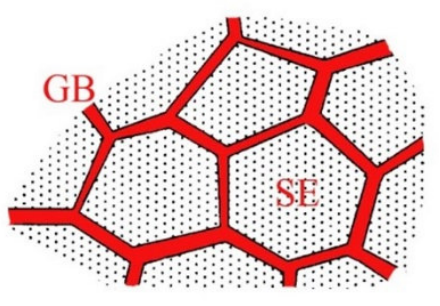

Figure 3 A 3D-net of FIT-pathways for solid-state ionic conductors on the different scale levels, (a) atomic scale: AdSIC $\alpha$-Agl; (b) mesoscopic scale: ordered domain structure in SIC (FIT- pathways are lines)

(c) microstructure scale: 3D-net of GBs with higher $\sigma_{i}$ along GB in solid electrolyte

Different levels of such 3D-network organization are possible (Figure 3): (a) atomic scale, i.e. inside unit cell of AdSIC; (b) mezo-scale as an ordered "domain structure" with additional FIT-pathways (domain boundaries) forming a 3D-network of FIT- pathways (e.g., the $\mathrm{Li}_{0.33} \mathrm{La}_{0.56} \mathrm{TiO}_{3} \mathrm{SIC}$ structure with the highest $\sigma_{\mathrm{i}}$ among the oxide ionic systems [13]); (c) microstructure scale as a 3D-network of grain boundaries (GBs) with a higher $\sigma_{i}$ along GB (as compared with the bulk) making the ion-transport characteristics of the system much higher. Such properties of GBs are usually characteristic of materials with rather low bulk $\sigma_{\mathrm{i}}$.

The interfacial design for many types of materials is in the focus of attention of many researches [1,2,4,11-15]. However, influence of homo- and heterophase boundaries with different structures on blocking or enhancing $\sigma_{\mathrm{i}}$ in various classes of ionic conductors is now the open and debatable question [14]. For example, GBs in high purity AdSICs polycrystals (with micron sized grains) practically do not influence on $\sigma_{i}$ (absence of the universal dynamic response) in large frequency interval. Evidently, processes of structural self-organization and formation of low-energy coherent GBs [13] with shallow potential landscape in the GB's area is a characteristic property of AdSICs. The coherency for AdSICs means not only a continuity of crystallographic planes across GB, but also preservation of FIT- tunnels [2].

As shown, the 3D network of topologically uniform FIT-tunnels (formed by conjugated by faces tetrahedrons) exists in AdSIC's structure on the level of the unit cell. It seems the determination of transitivity from unit cells of periodic crystallographic groups and nets of interstitial sites \{polyhedral\} inside them to structure description of unit cells as a rod (cylinder) packing [15], where rods are FIT tunnels can serve as an effective way for the selection and design of new high-performance ionic materials.

\section{CONCLUSION}

Currently, the materials science, using methods of generalized crystallography, becomes more and more mathematically focused. Transition to the nano-world leads inevitably to the necessity of combining various structural units and modules in 3D space. For example, chains and rings of atomic tetrahedrons conjugated by faces are observed in crystal-like and liquid-like, inorganic and organic ionic materials, denoting their stability. This can give extremely ordered, but not necessary periodic structures. Analysis of such configurations is based on ideas of "new geometry" [15]. It allows approaching the answer to the question: 
"how the long-range order (not necessarily periodicity) evolving from local principles of atomic ordering", changes our insights into phase transitions and opens "new nature codes".

In this work, a new geometry of dense packing of FIT-tunnels in advanced superionic conductors has been revealed. The main descriptor of AdSIC's structure is a 3D -percolation network of uniform low energy FITtunnels inside elementary cell of a rigid lattice of immobile ions. Each FIT-tunnel presents a chain of atomic tetrahedrons conjugated by faces, where tetrahedrons are interstitial low energy T-sites through which mobile ions move. The anomaly in frequency behavior of $\sigma_{\mathrm{i}}$ in AdSIC polycrystals compared with other solid-state ionic conductors is explained based on theory of fast ionic transport in non-uniform potential landscape (SDA). The deep understanding of the structure and properties of AdSICs is a guide for purposeful searching and designing new solid-state ionic materials and devices.

\section{ACKNOWLEDGEMENTS}

This research was financially supported by the State Task No. 075-00475-19-00.

\section{REFERENCES}

[1] DESPOTULI, A.L. and ANDREEVA, A.V. Nanoionics: new materials and supercapacitors. Nanotechnol Russia. 2010. vol. 5, no. 7-8, pp. 506-520.

[2] ANDREEVA, A.V. and DESPOTULI, A.L. Interface design in nanosystems of advanced superionic conductors. lonics. 2005. vol. 11, no. 1-2, pp. 152-160.

[3] BINDI, L., EVAIN, M., PRADEL, A., ALBERT, S., RIBES, M. and MENCHETTI, S. Fast ion conduction character and ionic phase-transitions in disordered crystals: the complex case of the minerals of the pearceite -polybasite group. Phys. Chem. Miner. 2006. vol. 33, no. 10, pp. 677-690.

[4] SUNANDANA, C.S. Introduction to solid state ionics: phenomenology and applications. Boca Raton-London- New York: CRC Press Taylor \& Francis Group, 2015. p.529.

[5] FLYGARE, W.H. and HUGGINS, R.A. Theory of ionic transport in crystallographic tunnels. J. Phys. Chem. Solids. 1973. vol. 34, no. 7, pp. 1199-1204.

[6] GELLER, S. Crystal structure of the solid electrolyte, RbAg4l5. Science. 1967. vol. 157 (3786), pp. 310-312.

[7] JONSCHER, A.K. The "universal" dielectric response. Nature 1977. vol. 267, pp. 673-679.

[8] DESPOTULI, A.L. and ANDREEVA, A.V. Maxwell displacement current and nature of Jonsher's dynamic response in nanoionics. Ionics. 2015. vol. 21, no. 2, pp. 459-469.

[9] FUNKE, K. Is there a "universal" explanation for the "universal" dynamic response? Berichte der Bunsengesellschaft für physikalische Chemie 1991. vol. 95, no. 9, pp. 955-964.

[10] WANG, Y., RICHARGS, W.D., ONG, S.P., MIARA, L.J., KIM, J.C., MO, Y. and CEDER, G. Design principles for solid-state lithium superionic conductors. Nat. mater. 2015. vol. 14, no. 10, pp. 1026 -1031.

[11] WANG, Y., RICHARDS, W.D., BO, S-H, MIARA, L., and CEDER G. Computational prediction and evaluation of solid-state sodium superionic conductors. Chem. Mater. 2017. vol. 29, no. 17, pp. 7475-7482.

[12] MA, C., CHENG, Y., CHEN, K., LI, J., SUMPTER, B.G., NAN, C-W., MORE, K.L., DUDNEY, N.J. and CHI, M. Mesoscopic Framework Enables Facile lonic Transport in Solid Electrolytes for Li-lon Batteries. Advanced Energy Materials. 2016. vol. 6, no. 11, 1600053.

[13] ANDREEVA, A.V. Interface design and processes of self-organization in nanosystems. J. Guangdong NonFerrous Metals. 2005. vol. 2-3, no. 1, pp. 244-250.

[14] HOOD, Z.D and CHI, M. Mechanistic understanding and strategies to design interfaces of solid electrolytes: insight gained from transmission electron microscopy. J.Mater.Sci. 2019. vol. 54, no. 5, pp. 10571-10594.

[15] LORD, E.A, MACKAY, A.L. and RANGANATHAN, S. New geometries for new materials. 2006. Cambridge: University press, p. 238. 